\title{
Directed Hierarchical Patterning of Polycarbonate Bisphenol A Glass Surface along Predictable Sites
}

\author{
Mazen Khaled \\ Department of Chemistry, King Fahd University of Petroleum \& Minerals, Dhahran 31261, Saudi Arabia \\ Correspondence should be addressed to Mazen Khaled; mkhaled@kfupm.edu.sa
}

Received 17 April 2015; Revised 29 July 2015; Accepted 29 July 2015

Academic Editor: Young Min Song

Copyright (C) 2015 Mazen Khaled. This is an open access article distributed under the Creative Commons Attribution License, which permits unrestricted use, distribution, and reproduction in any medium, provided the original work is properly cited.

\begin{abstract}
This paper reports a new approach in designing textured and hierarchical surfaces on polycarbonate bisphenol A type glass to improve hydrophobicity and dust repellent application for solar panels. Solvent- and vapor-induced crystallization of thermoplastic glass polycarbonate bisphenol A (PC) is carried out to create hierarchically structured surfaces. In this approach dichloromethane (DCM) and acetone are used in sequence. Samples are initially immersed in DCM liquid to generate nanopores, followed by exposing to acetone vapor resulting in the generation of hierarchical structure along the interporous sites. The effects of exposure time on the size, density, and distance of the generated spherules and gaps are studied and correlated with the optical transmittance and contact angle measurements at the surface. At optimized exposure time a contact angle of $98^{\circ}$ was achieved with $80 \%$ optical transmittance. To further increase the hydrophobicity while maintaining optical properties, the hierarchical surfaces were coated with a transparent composite of tetraethyl orthosilicate as precursor and hexamethyldisilazane as silylation agent resulting in an average contact angle of $135.8^{\circ}$ and transmittance of around 70\%. FTIR and AFM characterization techniques are employed to study the composition and morphology of the generated surfaces.
\end{abstract}

\section{Introduction}

Large number of studies have been performed to develop different techniques to design and produce hydrophobic surfaces to mimic self-cleaning plants such as the Lotus leaf by controlling the surface topography and chemistry [1-4]. The self-cleaning properties developed from the fact that the Lotus leaf surface has a hierarchical structure made of micro- and nanopatterns resulting in low adhesion forces of the water droplet which aid in the droplet movement. Several crop plants also have the same characteristics like the Lotus leave, for example, Brassica, Alchemilla, and Lupinus [5-7]. Mimicking the nature and generating surface hydrophobicity improve the performance of the photovoltaic devices by minimizing the dust accumulation at the surface which result in the degradation of material and significant decrease in light transmittance. Polycarbonate glass, bisphenol A type, is one of the promising materials to be modified to develop optimum hydrophobicity/transmittivity relationship due to the relatively facile chemical modification using appropriate solvents. Polycarbonate (PC) glass, one of the lowest cost materials, is used as protective covers for photovoltaic (PV) panels due to its high mechanical flexibility and low density. Many papers reported the surface texturing of polycarbonate glass at micro/nanoscales to generate a hydrophobic texture $[8,9]$. The resulting hydrophobic texture enhances the nonwetting properties by increasing the trapped air between the surface texture posts. This, in turn, leads to a superhydrophobic behavior of the textured surface, since liquid droplets lay on the air pockets.

Many attempts were carried out to generate superhydrophobic surfaces of polycarbonate glass by controlling surface morphology; however, all these approaches either are technically limited such as lithography or may result in the desired micro/nanoarchitecture but at the expense of optical properties. Among the promising solvents with suitable PC interactivity is acetone. Acetone-induced crystallization was studied extensively in the recent decade [10-12]; however, the fast dynamic of acetone crystallization results in the development of hierarchical spherules with high aspect ratio on the surface which reduce the glass transmittance dramatically and hence reduce their applicability in photovoltaic panels. 
Varanasi's group generated a superhydrophobic surface by immersing PC into acetone at different amounts of time [13]. However, a long immersion period (i.e., $30 \mathrm{~min}$ ) was required to create a superhydrophobic surface, which introduces the possibility of deformation of the PC plate or the formation of powder on the surface by the recrystallization of PC after solvent evaporation and the significant reduction in optical properties. Recently, Go et al. [14] tried different organic solvents such as $\mathrm{CH}_{2} \mathrm{Cl}_{2}$, methyl ethyl ketone, ethyl acetate, toluene, and isopropyl alcohol and found that only $\mathrm{CH}_{2} \mathrm{Cl}_{2}$ generated nanoporous structures in a short period ( $5 \mathrm{~s})$. They further silanize the surface to yield a superhydrophobic surface with contact angle of $161^{\circ}$. According to our experimental results the $5 \mathrm{~s}$ immersion in dichloromethane reduces the optical transmittance significantly. Therefore, in this paper a new approach is reported to control the generation and growth of the spherules as well as intrapores distance while maintaining significant transmittance and further improve the hydrophobicity by silanizing the surface using sol gel technique. This method is economic and facile and includes the immersing of PC glass in dichloromethane for very short time to generate nanopores followed by exposing the same surface to acetone vapor directed towards the interporous sites to generate the hierarchical structures while maintaining partially unaffected surfaces between spherules to minimize the reduction of the optical properties. The effects of different dipping and vapor exposure periods on the surface morphology and wettability of the textured surfaces were studied. Commercially available PC plate from Juliet was chosen to prepare the superhydrophobic surfaces using the simple dipping and drying process. The generated PC surfaces were studied by UV-Vis, FTIR, and AFM and by measuring the contact angle between the surface and water droplets.

\section{Materials and Methods}

Bisphenol A polycarbonate sheets [2,2-bis(p-hydroxyphenyl) propane] of $3 \mathrm{~mm}$ thickness made by Juliet Company were used. Solvents used were reagent grade and acquired from Sigma-Aldrich. Dichloromethane puriss. p.a., ACS reagent, reag. ISO, $\geq 99.9 \%$ (GC) (Sigma-Aldrich), was used to initiate the nanopores. Acetone solvent from Sigma-Aldrich (purity $\geq 99.5 \%$, b.p.: $56^{\circ} \mathrm{C}$, MW: 58.08 , and v.p.: $184 \mathrm{mmHg}$ at $20^{\circ} \mathrm{C}$ ) was used for the vapor-induced crystallization process. Tetraethoxysilane (TEOS) and 1,1,1,3,3,3-hexamethyldisilazane (HMDS) were purchased from Sigma-Aldrich and used in the sol gel method to generate a superhydrophobic coating on PC glass.

The bisphenol A 2,2-bis(p-hydroxyphenyl) based polycarbonate plates were cleaned with soap and then sonicated for $15 \mathrm{~min}$ in a mixture of isopropyl alcohol and deionized water. The cleaned samples were left to dry at ambient conditions for few hours and then immersed in dichloromethane solvent for few seconds at room temperature. The samples were then removed from the solvent and immediately immersed in water. Subsequently, the PC samples were exposed to acetone vapor passing through a $1 \mathrm{~cm} \times 1 \mathrm{~cm}$ squared outlet, for 1,2 , and 4 minutes where the liquid acetone temperature was maintained at $33^{\circ} \mathrm{C}$.
A $25 \mathrm{~mL}$ cylindrical glass is used in the setup which evaporate acetone in the vertical direction towards the PC surface. After exposure to acetone vapor the polycarbonate sheet is removed, cleaned with water, and left to dry at ambient room temperature. The exposure time plays a crucial role in the polycarbonate crystallization process. Thus, polycarbonate sheets are exposed to acetone vapor for different periods of time in order to investigate the effect of time on the surface texture characteristics, such as size, density, and distance of the generated spherules and gaps.

2.1. TEOS/HMDS Sol Gel Preparation. The $\mathrm{pH}$ value for deionized water was adjusted to 0.5 by adding $4-5$ drops of $\mathrm{HCl}$ solution. Tetraethyl orthosilicate (TEOS) was hydrolyzed with acidic deionized water at fixed molar ratio of ITEOS : 48 $\mathrm{C}_{2} \mathrm{H}_{5} \mathrm{OH}: 4 \mathrm{H}_{2} \mathrm{O}(\mathrm{pH} 0.5)$ at $70^{\circ} \mathrm{C}$ with vigorous stirring for $2 \mathrm{~h}$ [11] and then adding the 1,1,1,3,3,3-hexamethyldisilazane (HMDS) $($ HMDS/TEOS $=2$ ) by adding the HMDS into 20 parts (1 drop at a time). The sequential addition of HMDS proceeded for $3 \mathrm{~h}$. Upon the fourth or fifth addition of HMDS and sonication of the sol, part of the sol transformed into transparent jelly. Adding HMDS is halted, and the transparent jelly retransformed into transparent sol when additional $\mathrm{C}_{2} \mathrm{H}_{5} \mathrm{OH}\left(\mathrm{C}_{2} \mathrm{H}_{5} \mathrm{OH} / \mathrm{TEOS}=24\right)$ was added for $1-2 \mathrm{~h}$. Besides the fixed $3 \mathrm{~h}$, this period of $1-2 \mathrm{~h}$ was required to break up the transparent jelly. Then, HMDS continued to be added until the end of the procedure. The transparent jelly was then no longer present. To obtain an ideal superhydrophobic sol gel, while maintaining transparency as high as possible, further $\mathrm{C}_{2} \mathrm{H}_{5} \mathrm{OH}\left(\mathrm{C}_{2} \mathrm{H}_{5} \mathrm{OH} / \mathrm{TEOS}=6\right)$ was added to the sol gel at the end of the addition of HMDS. Then, the sol gel was aged in a refrigerator at $20^{\circ} \mathrm{C}$ for $72 \mathrm{~h}$. The sol gel was dip-coated onto the polycarbonate substrate for $5 \mathrm{~s}$. Heat treatment at $200^{\circ} \mathrm{C}$ for $2 \mathrm{~h}$ was used to repel the excess ethanol and stabilize the film.

Atomic Force Microscope (AFM) (5100-Agilent Technologies) was used for scan and for characterizing the surface topography and roughness profile in contact. Image analysis was performed using software WSxM v5.0 Develop 6.2. Silicon nitride probes tip was used with radius in the range of $20-60 \mathrm{~nm}$ and specified force constant $(k)$ of $0.12 \mathrm{~N} / \mathrm{m}$ manufactured by Bruker AFM Probes Co. Fourier-Transform Infrared (FTIR) (Thermo Electron Corp.) was utilized in the identification of different functional groups that are present on the surface before and after the texturing process. UV-Vis spectrophotometer (PerkinElmer Co.) was utilized to measure the transmittance of the polycarbonate sheet before and after the patterning process. A Goniometer (KYOWA DM701 ) is used to determine the degree of both the hydrophilicity and the hydrophobicity of the surface by measuring the static contact angles between a deionized water droplet (of $0.4-5 \mu \mathrm{L}$ volume) and the surface.

\section{Results and Discussion}

Patterned nanoporous structures were first generated by dipping PC in dichloromethane and then exposure to acetone vapor for different durations. It was found that the dipping duration is very critical for maintaining the optical properties 


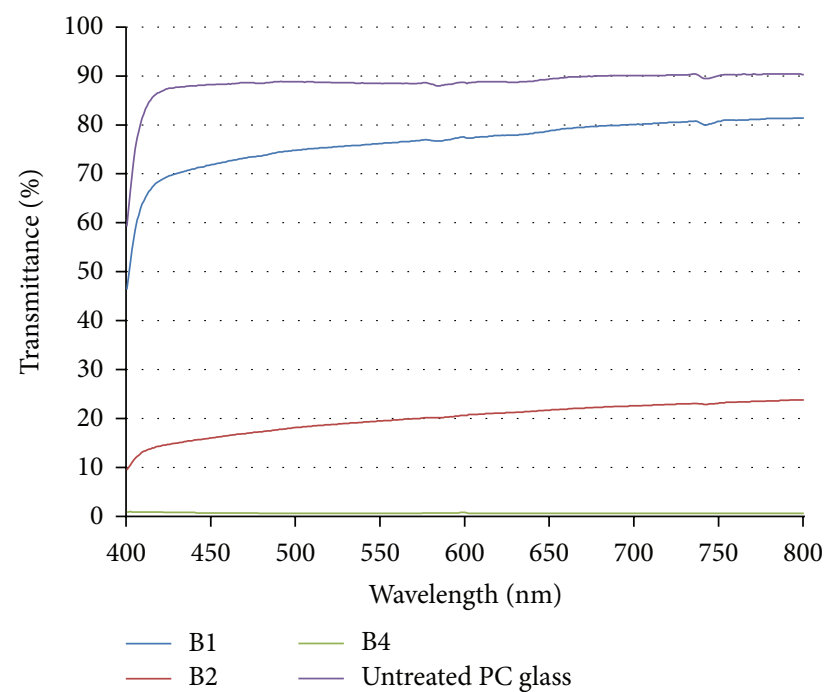

FIGURE 1: \% transmittance of untreated PC glass and after treatment with dichloromethane: B1 for $1 \mathrm{~s}, \mathrm{~B} 2$ for $2 \mathrm{~s}$, and B4 for $4 \mathrm{~s}$.

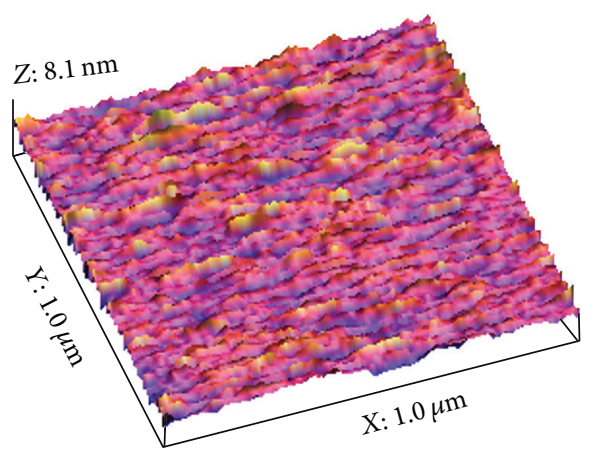

FIGURE 2: 3D AFM topography image of untreated polycarbonate glass showing a smooth surface.

of the material to be useful in the photovoltaic solar panels. Different periods of immersion in dichloromethane were carried out and it was found that after $2 \mathrm{~s}$ immersion the PC glass maintains $80 \%$ transmittance as in Figure 1 and drops drastically after that which is in agreement with results from [14]. Therefore, a $2 \mathrm{~s}$ immersion duration in DCM is considered for all the experiments. The AFM of untreated PC glass surface after ultrasonication for 15 minutes in isopropyl alcohol, in Figure 2, shows a smooth surface with very low surface roughness as noted from the $z$ scale.

AFM scans were carried out on PC immersed in DCM for $2 \mathrm{~s}$ up to $5 \mathrm{~s}$. Figure 3(a) shows the 3D AFM image of polycarbonate glass after immersing in dichloromethane for $2 \mathrm{~s}$. It can be noted that the surface shows a nonuniform nanoporous morphology with unaffected areas in between. A line profile was taken through Figure 3(a) across pores and shown in Figure 3(b) to obtain quantitative information of the topographical details. The line profile shows main pores with depth in the range of $10-12 \mathrm{~nm}$ and maximum surface width of $2 \mu \mathrm{m}$. The distance between the pores is in the range of $4 \mu \mathrm{m}$. The line profile also shows the initiation of infant pores with smaller widths and shallower depths. The resulting contact angle was $91.8^{\circ}$ compared to $77.6^{\circ}$ for the untreated PC glass as shown in Figure 4. The transmittance of the PC glass immersed in DCM was $88 \%$ compared to $90 \%$ for the untreated PC glass. It was noted that beyond $2 \mathrm{~s}$ the PC transmittance decreases significantly due to the development of large micropores as shown in the AFM image in Figure 3(c).

In order to generate hierarchical structures, a series of experiments were carried out on different PC specimen, after immersion for $2 \mathrm{~s}$ in DCM, at different exposure time periods to acetone vapors. The acetone source was heated gently at $33^{\circ} \mathrm{C}$ to enhance evaporation. Acetone-induced crystallization of polycarbonate glass was studied extensively [15-21]. Accordingly, nucleation occurred at the initial stage of vapor-induced crystallization and poor crystals become perfect through segment reorganization with increasing exposure time and spherulites' growth at elongated periods of exposure. The generation of the spherules with needlelike structure on top is related back to a molecular or atomic rearrangement process that takes place in order to achieve stable orientations, which is termed a crystallization process. High spherules density increases drastically the surface roughness and hydrophobicity at the expense of the optical properties. Large number of spherules that cover the whole surface to improve its hydrophobicity will reduce the surface transmittance and hence limit its applicability to PV panels. Therefore, acetone vapors are expected to reduce the crystallization dynamic time since much less acetone is available at the surface which prevent absorption by the surface layers compared to liquid acetone. Figures 5(a) and 5(b) show the AFM of the PC after immersion for $2 \mathrm{~s}$ in dichloromethane and exposure for 1 and 2 minutes to acetone vapors. At the first stage (Figure 5(a)), the spherules are still small and did not expand vertically significantly yet. However, after $2 \mathrm{~min}$ exposure complete micrometer size spherules are observed as in the AFM topographic Figure 5(b) with fine nanostructures on top as noted in the line profile in Figure 5(c). The surface morphology further reveals the presence of areas of high posts followed by relatively unaffected void areas which close up at the 2-minute exposure limit.

Voids between the spherules are necessary to allow the surface to maintain sufficient optical transmittance.

Increasing exposure time of PC for the acetone vapors will cause an increase in the spherules size, hence reducing the path for light transmittance. Figure 6 shows the AFM image of PC glass after $4 \mathrm{~min}$ exposure to acetone vapor. It can be noticed that the spherules are closing up while no significant change in spherule height is observed as compared to Figure 5(b). The optical transmittance as shown in Figure 1 was $80 \%$ for the $1 \mathrm{~min}$ exposure to acetone vapors versus $26 \%$ for the $2 \mathrm{~min}$ exposure to acetone vapors. After $4 \mathrm{~min}$ of treatment with acetone vapors the optical transmittance was reduced to few percent and the resulting PC surface had a contact angle of $98^{\circ}$.

The contact angles for the $1 \mathrm{~min}$ and 2 min samples exposed to acetone vapors were $80.3^{\circ}$ and $82.1^{\circ}$, respectively, as shown in Figure 7. Hence, careful selection of experimental conditions is required to generate the desired 


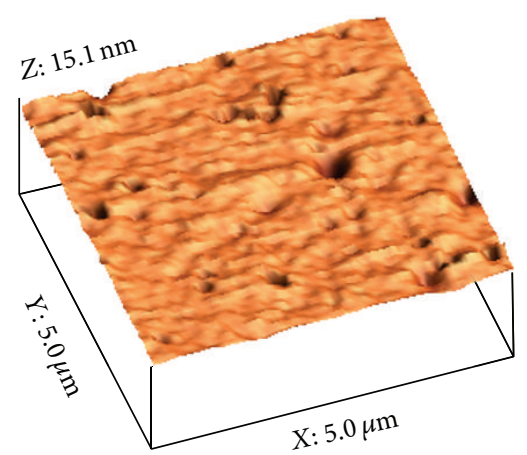

(a)

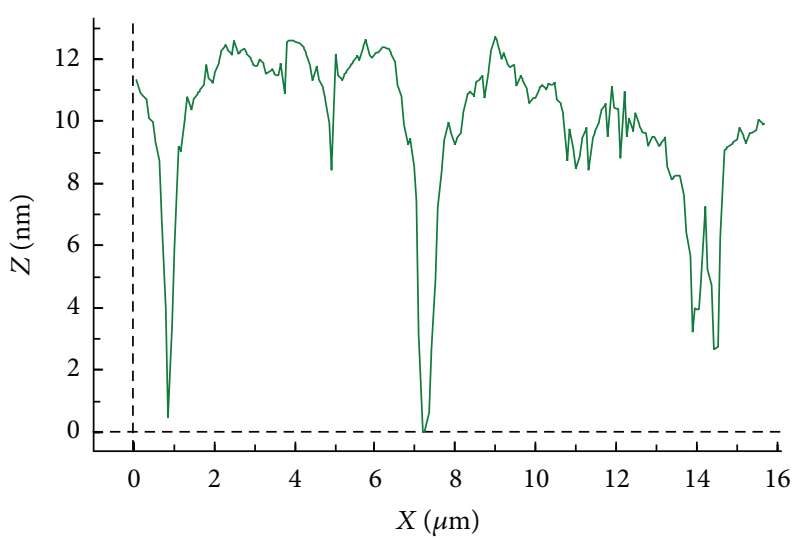

(b)

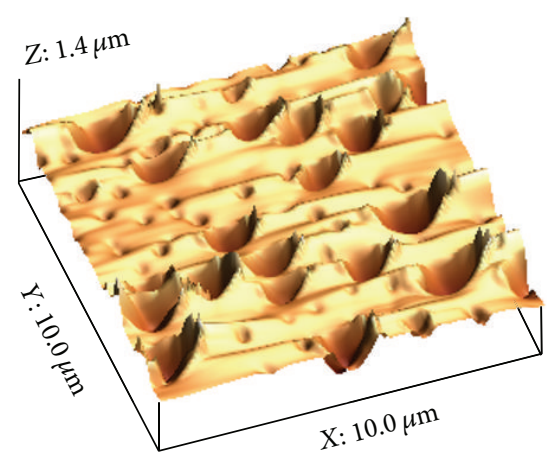

(c)

FIGURE 3: AFM topography image of PC immersed in DCM for (a) $2 \mathrm{~s}$ and (b) line profile and (c) $5 \mathrm{~s}$.

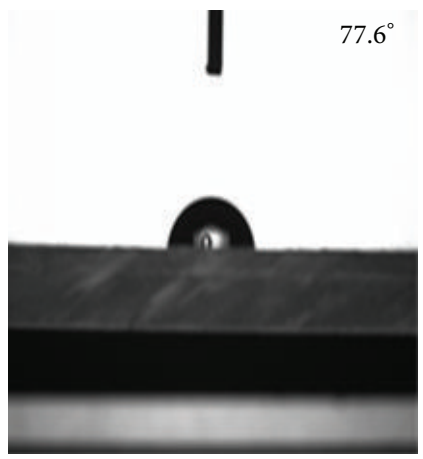

(a)

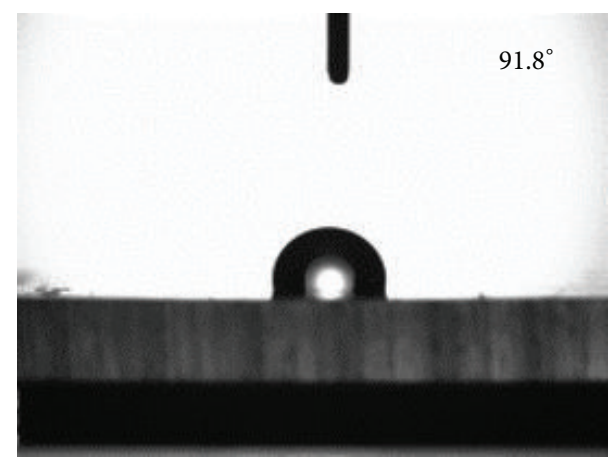

(b)

FIgURE 4: Contact angle of PC before (a) and after $2 \mathrm{~s}$ immersion in dichloromethane (b).

hierarchical architecture without imposing significant losses on the optical transmittance. Therefore, the $1 \mathrm{~min}$ sample treated sample was selected for sol gel coating to improve further its hydrophobicity.

FTIR was carried out on all textured surfaces as shown in Figure 8. The spectra are identical except for the decrease of the carbonyl peak intensities around $1766 \mathrm{~cm}^{-1}$ indicating the phase transformation of the polymer from the amorphous phase, which allows a higher degree of freedom to the chains motion and bonds vibrations, to the crystalline phase, in which the molecules are tightly packed and consequently the vibrations and motions are restricted [10, 15, 21]. It also indicates that the acetone interaction with the polycarbonate is a physical process as there is no new peak emerged after the treatment process [15].

Sol gel coating is very well known to improve surface properties of different materials. For photovoltaic cells applications the interest is in developing a superhydrophobic coating while maintaining high optical transmittance. The equation for the hydrolysis of TEOS is shown in Scheme 1. 


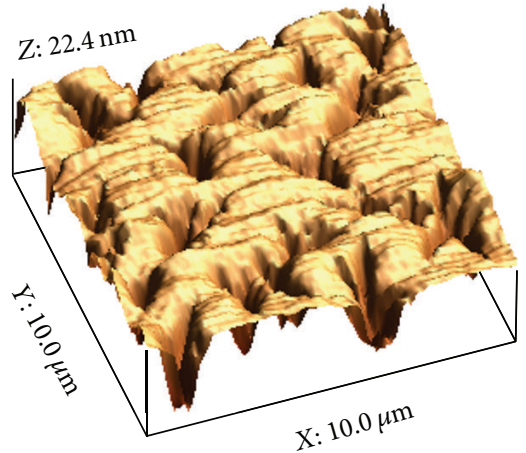

(a)

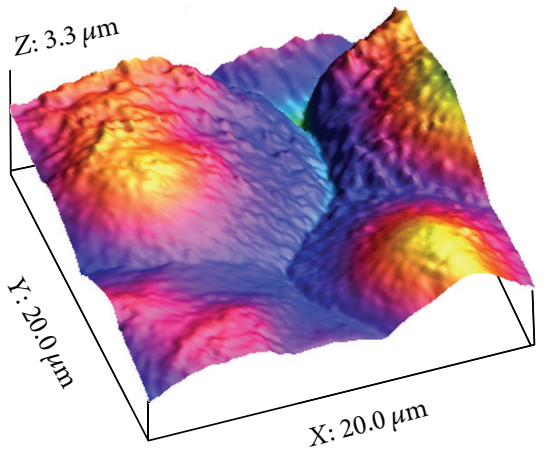

(b)

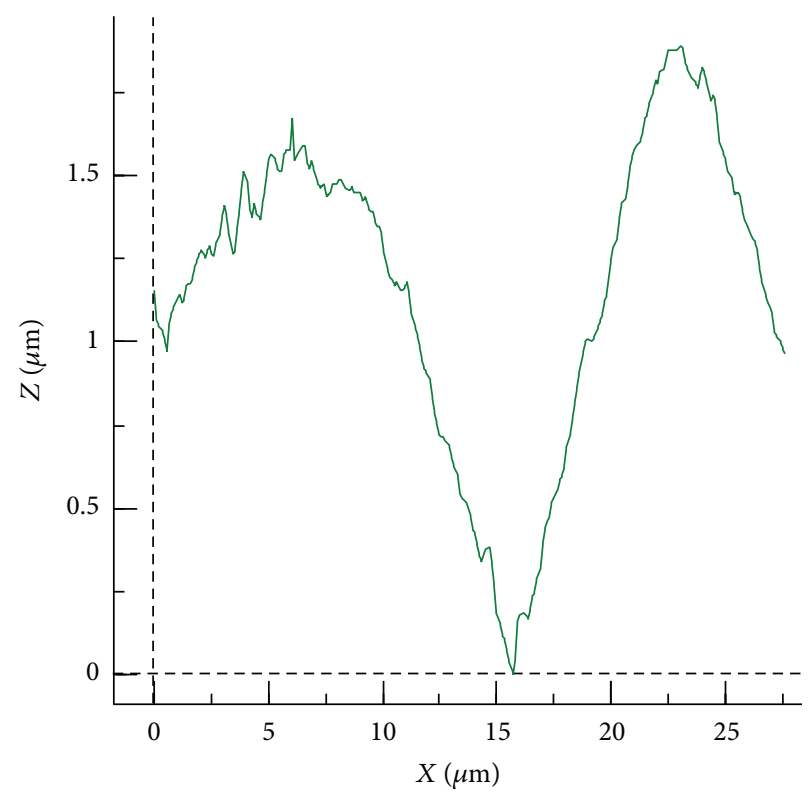

(c)

FIGURE 5: AFM image of PC after immersion for $2 \mathrm{~s}$ in dichloromethane and exposure to acetone vapors for (a) $1 \mathrm{~min}$ and (b) 2 min; (c) line profile of (b).

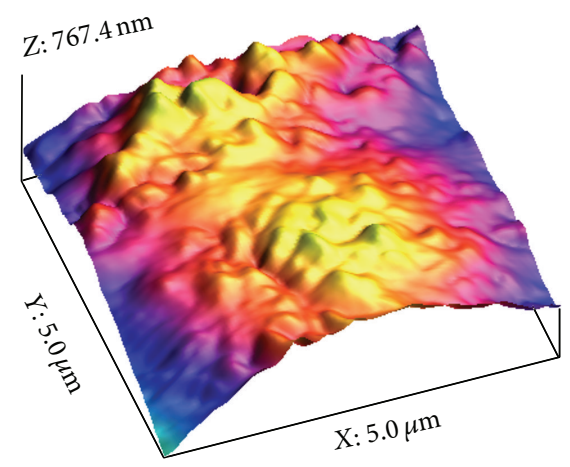

Figure 6: AFM image of PC glass after 4 min exposure to acetone vapor: (a) 3D, (b) 2D, and (c) line profile.

The equation for the reaction between $\mathrm{Si}-\mathrm{OH}$ and HMDS to form the hydrophobic bond is shown in Scheme 2.
After coating with sol gel it was found that the contact angle increased significantly to $135.8^{\circ}$ as shown in Figure 9. The optical transmittance of the DCM/acetone vapor/sol gel coated polycarbonate glass sample was found to be around $70 \%$ as shown in Figure 10.

\section{Conclusion}

In conclusion a facile method was developed using the solvent-induced crystallization properties of a polycarbonate surface by using in sequence the immersion of polycarbonate sheet in dichloromethane and followed by exposure to acetone vapor which resulted in a hierarchically structured surface. The optimum exposure conditions were found to be $2 \mathrm{~s}$ immersion in dichloromethane and exposure for 1 minute to acetone vapors yielding $80 \%$ optical transmittance. FT-IR data indicated the strong crystallization, induced with the acetone vapor, by the large shift in the peaks intensity. Sol gel 


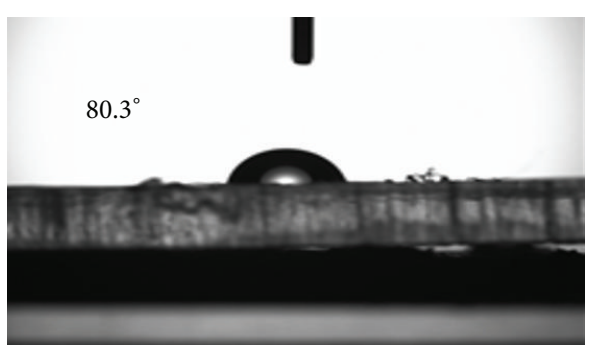

(a)

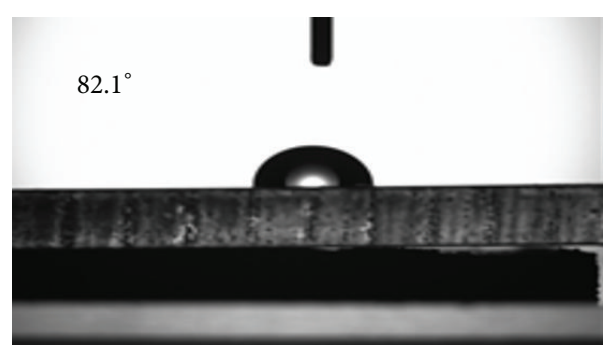

(b)

FIGURE 7: Contact angle of PC after $2 \mathrm{~s}$ immersion in dichloromethane and exposure to acetone vapors for (a) $1 \mathrm{~min}$ and (b) $2 \mathrm{~min}$.
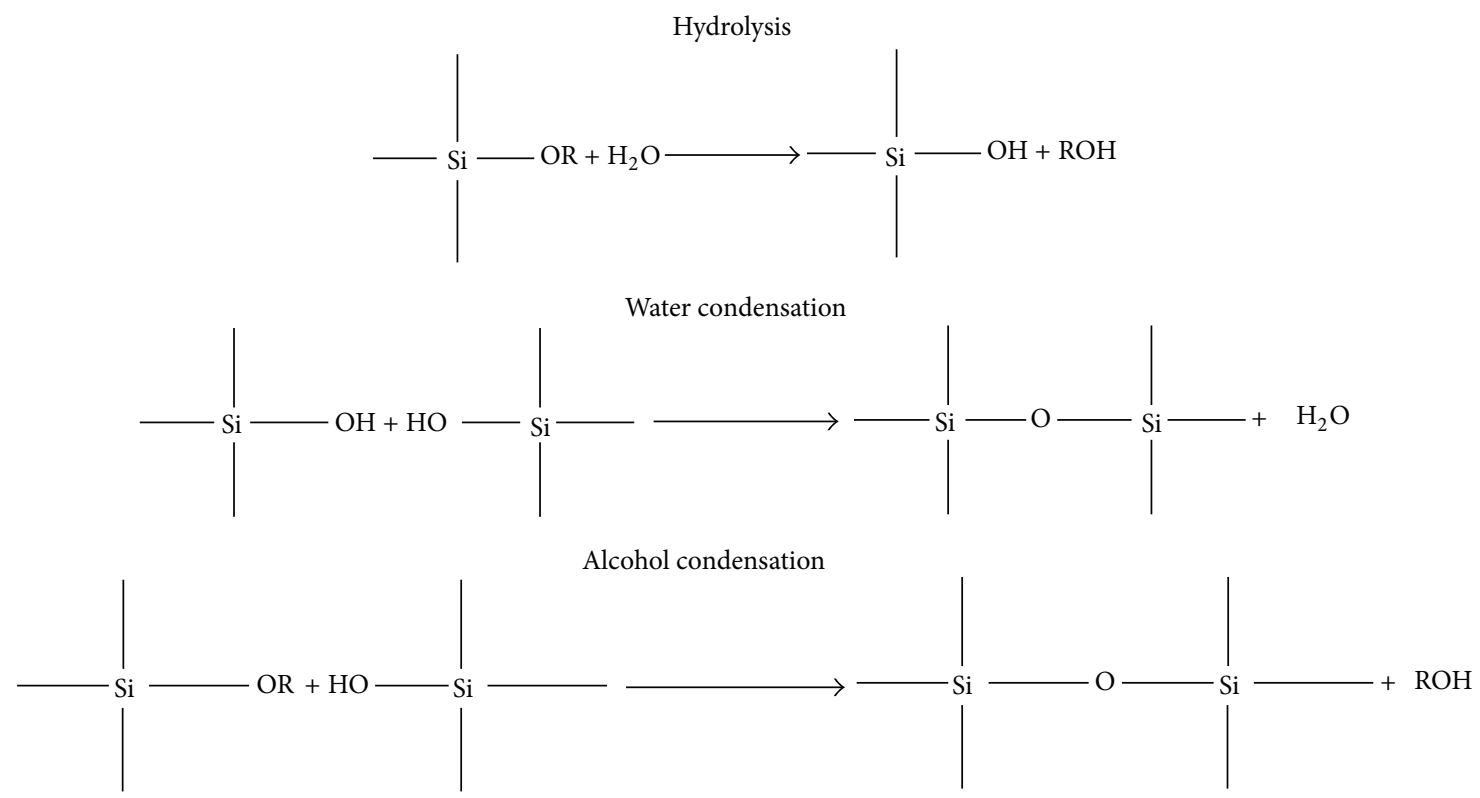

SCHEME 1

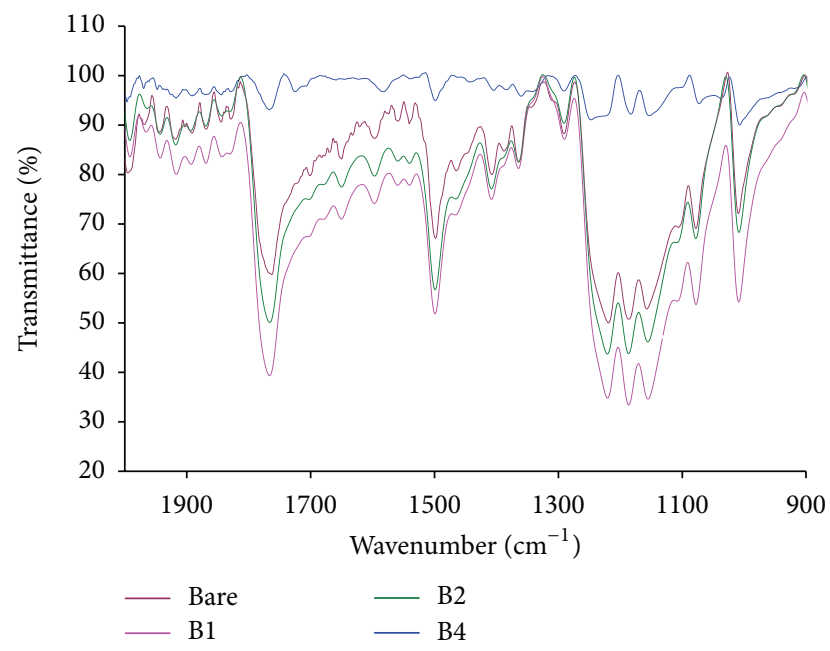

FIGURE 8: FT-IR spectra of all the textured polycarbonate surfaces where the burgundy graph corresponds to the untreated polycarbonate.

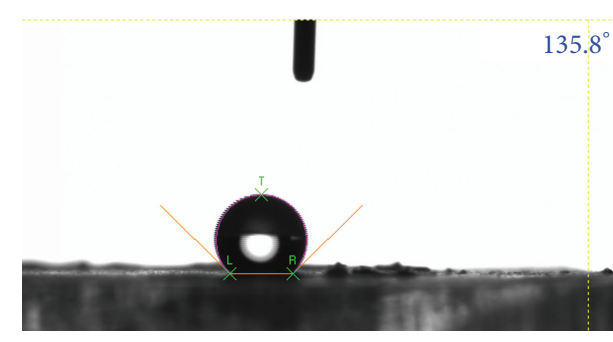

Figure 9: Contact angle of the sol gel dip-coated textured polycarbonate samples after immersion in pure liquid $\mathrm{CH}_{2} \mathrm{Cl}_{2}$ for $2 \mathrm{~s}$ and exposed to acetone vapor for $1 \mathrm{~min}$.

coating using TEOS/HMDS film after it had been textured by DCM (2s) and acetone vapor for 1 min exhibited a contact angle CA $135.8^{\circ}$. Hence, proper optimization of spherules growth, dimensions, and interspherules distances and then sol gel coating resulted in a significantly hydrophobic polycarbonate surface with optical transmittance around $70 \%$. This 


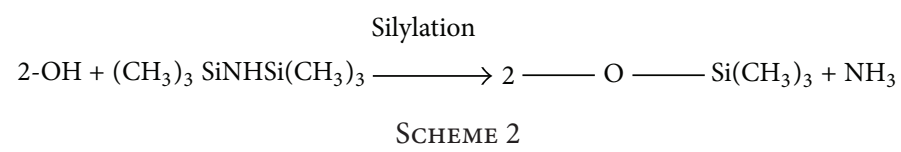

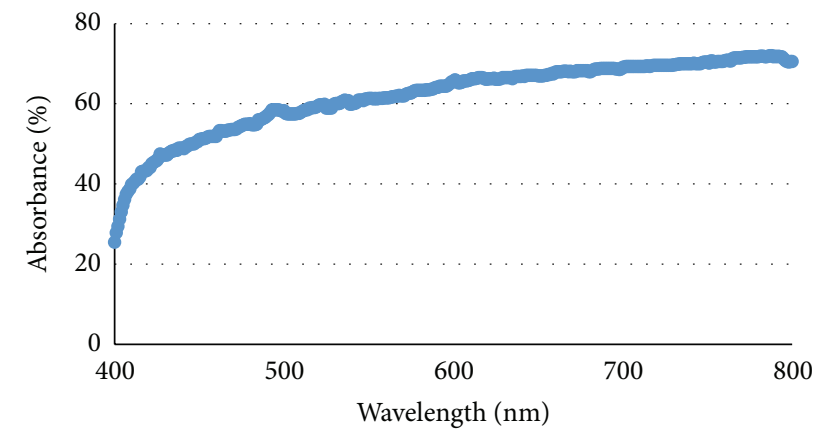

FIGURE 10: \% transmittance of PC glass and after treatment with $2 \mathrm{~s}$ dichloromethane, $1 \mathrm{~min}$ acetone vapor, and TEOS/HMDS sol gel coating.

approach is promising in developing future superhydrophobic, economic, and less technologically demanding surfaces for application as photovoltaic panels.

\section{Conflict of Interests}

The author declares that there is no conflict of interests regarding the publication of this paper.

\section{Acknowledgments}

This project was funded by the National Plan for Science, Technology and Innovation (MAARIFAH), King Abdulaziz City for Science and Technology, through the Science and Technology Unit at King Fahd University of Petroleum and Minerals (KFUPM), Kingdom of Saudi Arabia, Award no. 11ADV2134-04. The support from the Center of Excellence for Research Collaboration with MIT is also acknowledged for funding Projects nos. MIT11111-11112.

\section{References}

[1] S. Anand, K. Rykaczewski, S. B. Subramanyam, D. Beysens, and K. K. Varanasi, "How droplets nucleate and grow on liquids and liquid impregnated surfaces," Soft Matter, vol. 3, no. 1, pp. 69-80, 2015.

[2] Y. Yoon, D. Kim, and J. B. Lee, "Hierarchical micro/nano structures for super-hydrophobic surfaces and super-lyophobic surface against liquid metal," Micro and Nano Systems Letters, vol. 2, pp. 1-18, 2014.

[3] Y. Yoon, D.-W. Lee, and J.-B. Lee, "Fabrication of optically transparent PDMS artificial lotus leaf film using underexposed and underbaked photoresist mold," Journal of Microelectromechanical Systems, vol. 22, no. 5, pp. 1073-1080, 2013.

[4] B. Bhushan, Y. C. Jung, and K. Koch, "Micro-, nano- and hierarchical structures for superhydrophobicity, self-cleaning and low adhesion," Philosophical Transactions of the Royal Society A: Mathematical, Physical and Engineering Sciences, vol. 367, no. 1894, pp. 1631-1672, 2009.

[5] R. Wang, K. Hashimoto, A. Fujishima et al., "Light-induced amphiphilic surfaces," Nature, vol. 388, no. 6641, pp. 431-432, 1997.

[6] C. Neinhuis and W. Barthlott, "Characterization and distribution of water-repellent, self-cleaning plant surfaces," Annals of Botany, vol. 79, no. 6, pp. 667-677, 1997.

[7] T. Verho, J. T. Korhonen, L. Sainiemi et al., "Reversible switching between superhydrophobic states on a hierarchically structured surface," Proceedings of the National Academy of Sciences of the United States of America, vol. 109, no. 26, pp. 10210-10213, 2012.

[8] Z. Fan, C. Shu, Y. Yu, V. Zaporojtchenko, and F. Faupel, "Vaporinduced crystallization behavior of bisphenol-A polycarbonate," Polymer Engineering \& Science, vol. 46, no. 6, pp. 729-734, 2006.

[9] N. Zhao, L. Weng, X. Zhang, Q. Xie, X. Zhang, and J. Xu, "A lotus-leaf-like superhydrophobic surface prepared by solventinduced crystallization," ChemPhysChem, vol. 7, no. 4, pp. 824$827,2006$.

[10] E. Turska and H. Janeczek, "Liquid-induced crystallization of a bisphenol-A polycarbonate," Polymer, vol. 20, no. 7, pp. 855-858, 1979.

[11] S. M. Aharoni and N. S. Murthy, "Effects of solvent-induced crystallization on the amorphous phase of polycarbonate of bisphenol A," International Journal of Polymeric Materials and Polymeric Biomaterials, vol. 42, no. 3-4, pp. 275-283, 1998.

[12] D. Park and J.-W. Hong, "Solvent-induced crystallization and interaction parameter of the blends of bisphenol A polycarbonate and poly(phenyl methacrylate)," Polymer Journal, vol. 29, no. 12, pp. 970-974, 1997.

[13] Y. Cui, A. T. Paxson, K. M. Smyth, and K. K. Varanasi, "Hierarchical polymeric textures via solvent-induced phase transformation: a single-step production of large-area superhydrophobic surfaces," Colloids and Surfaces A: Physicochemical and Engineering Aspects, vol. 394, pp. 8-13, 2012.

[14] S. Go, M. Han, and Y. Ahn, "Formation of nanoporous polycarbonate surfaces and their chemical modification for superhydrophobicity," Bulletin of the Korean Chemical Society, vol. 33, no. 11, pp. 3899-3902, 2012.

[15] J. Brandrup, E. H. Immergut, E. A. Grulke, A. Abe, and D. R. Bloch, Polymer Handbook, Wiley, New York, NY, USA, 1999.

[16] L. H. Sperling, Introduction to Physical Polymer Science, John Wiley \& Sons, Hoboken, NJ, USA, 2005.

[17] F. L. B. O. de Oliveira, M. C. A. M. Leite, L. L. O. Couto, and T. R. Correia, "Study on bisphenol-A polycarbonates samples crystallized by acetone vapor induction," Polymer Bulletin, vol. 67, no. 6, pp. 1045-1057, 2011.

[18] R. P. Sheldon and P. R. Blakey, "Liquid-induced crystallization in polymers," Nature, vol. 195, no. 4837, pp. 172-173, 1962.

[19] G. G. Liang, W. D. Cook, H. J. Sautereau, and A. Tcharkhtchi, "Diallyl orthophthalate as a reactive plasticizer for polycarbonate. Part 1: uncured system," European Polymer Journal, vol. 44, no. 2, pp. 366-375, 2008. 
[20] E. Turska, W. Przygocki, and M. Masđowski, "Crystallization kinetics of polycarbonates. I," Journal of Polymer Science Part C: Polymer Symposia, vol. 16, no. 6, pp. 3373-3377, 1967.

[21] J. P. Mercier, G. Groeninckx, and M. Lesne, "Some aspects of vapor-induced crystallization of polycarbonate of bisphenol A," Journal of Polymer Science Part C: Polymer Symposia, vol. 16, no. 4, pp. 2059-2067, 1967. 

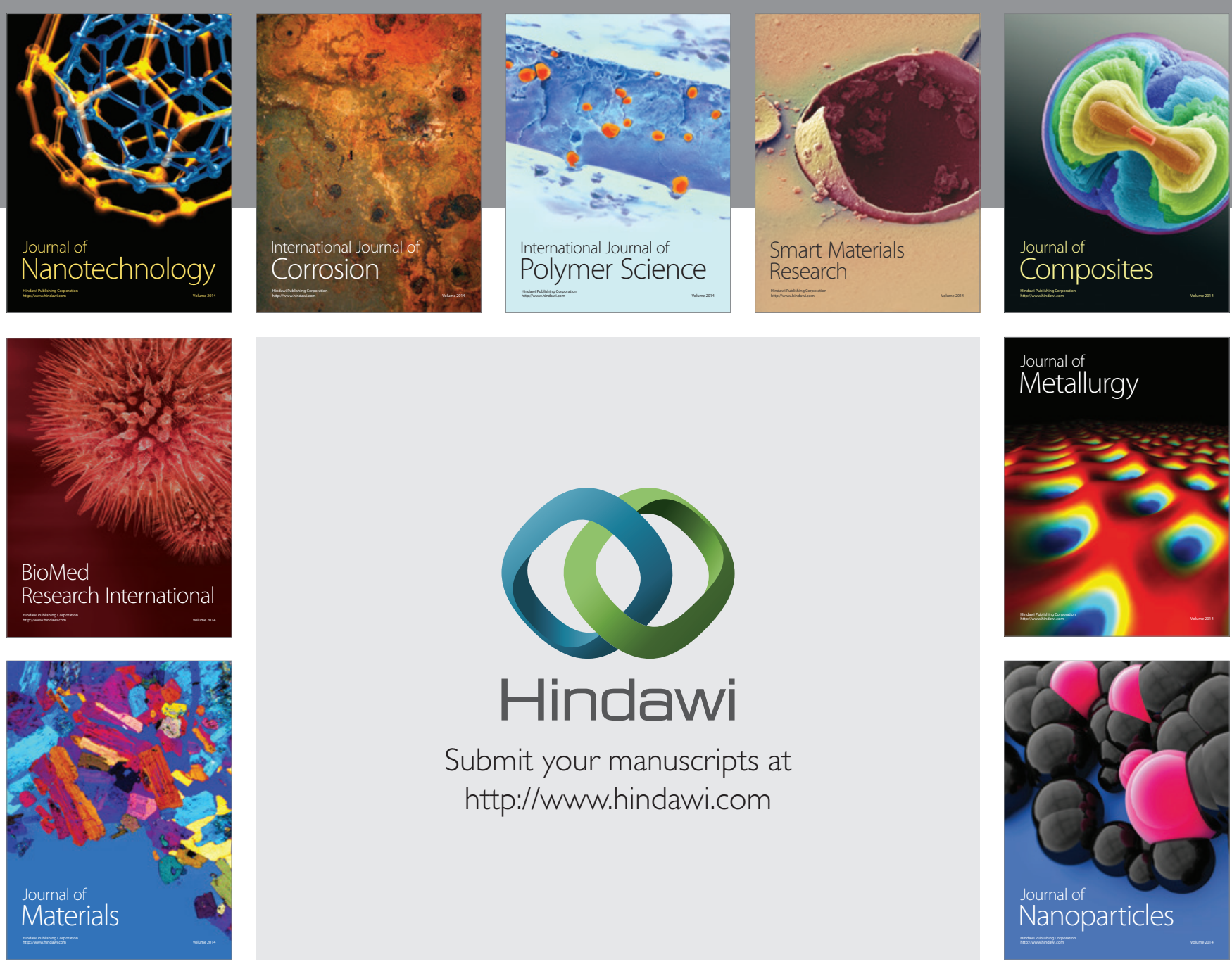

Submit your manuscripts at http://www.hindawi.com
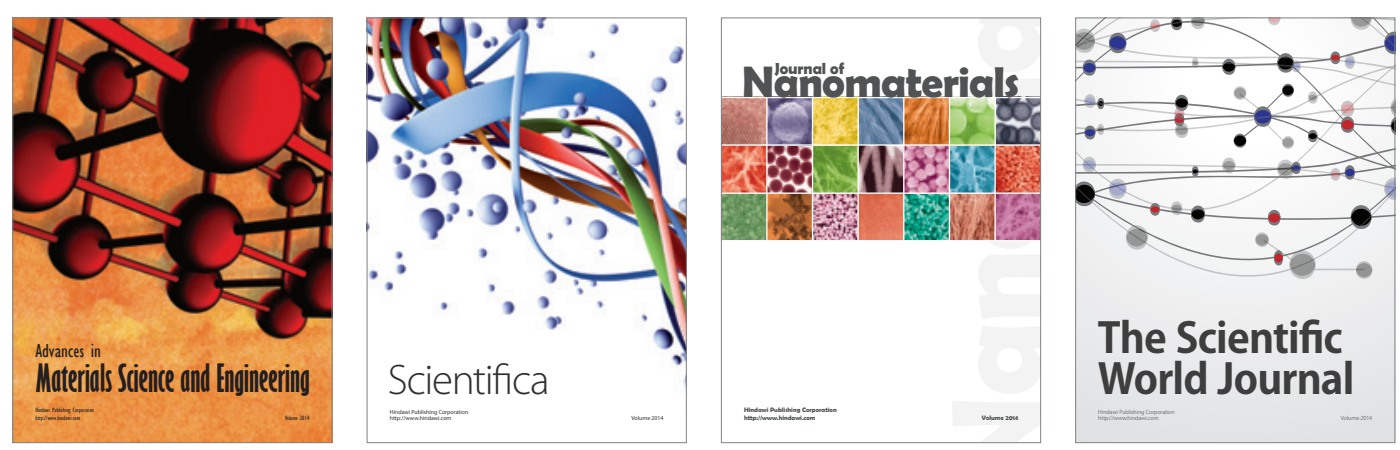

\section{The Scientific World Journal}
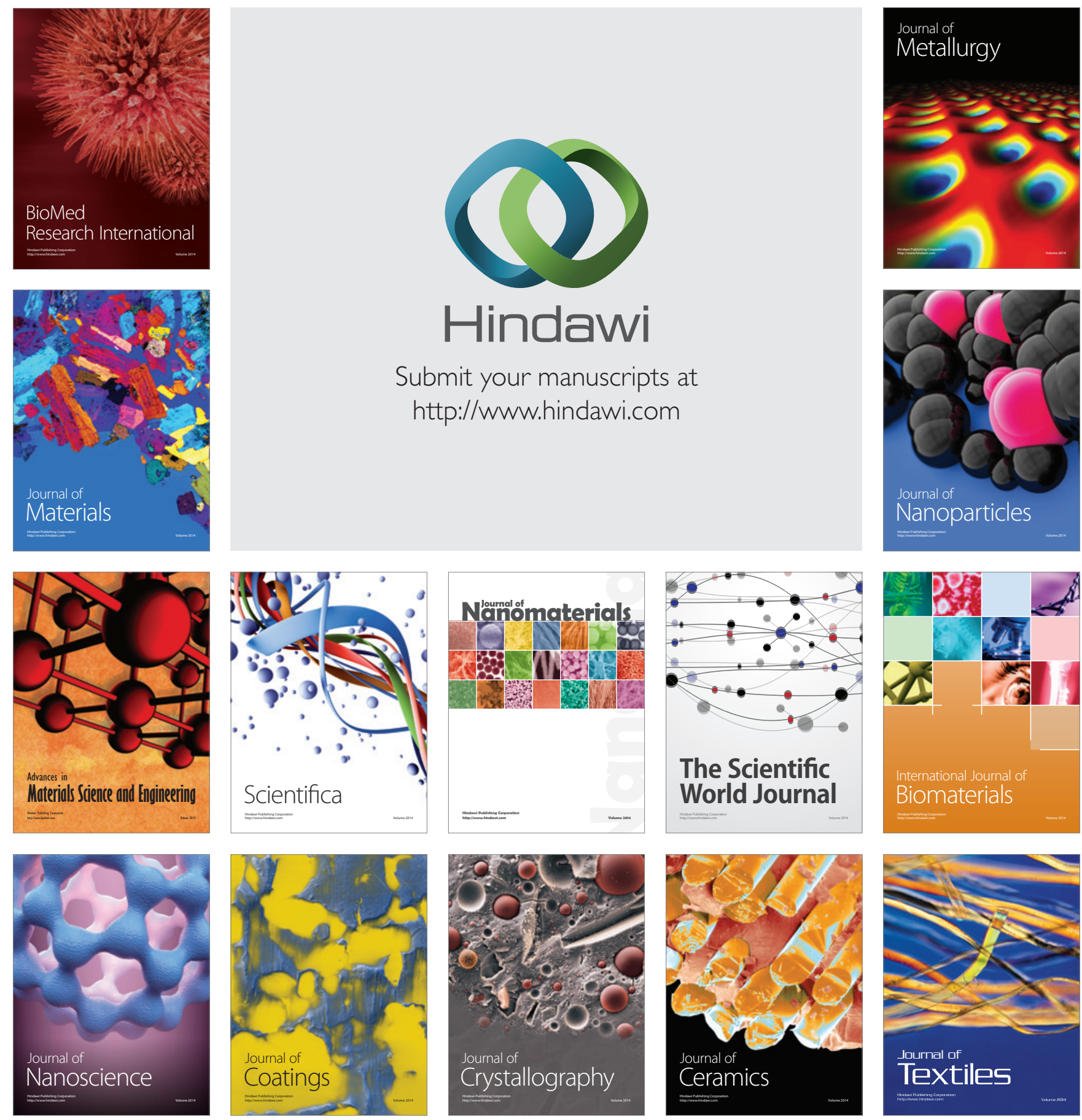